\title{
Retroperitoneal lymphadenopathy in familial Mediterranean
} fever

\section{David Rimon, Yoseph Meir and Leon Cohen}

Department of Internal Medicine B, Rheumatology Service and the Department of Radiology, Faculty of Medicine, Technion-Israel Institute of Technology, Lady Davis Carmel Hospital, Haifa, Israel.

\begin{abstract}
Summary: Peripheral lymphadenopathy is rarely observed, whereas mesenteric lymphadenopathy is found occasionally on laparotomies in patients with familial Mediterranean fever (FMF). Retroperitoneal lymphadenopathy was reported only once in an autopsy of a patient with FMF. Our case is the second one, and the first one to be diagnosed during life, by means of abdominal ultrasonography and computerized tomography. In patients with FMF, where lymph node biopsy was done, the pathological finding was non-specific lymphoid hyperplasia.
\end{abstract}

\section{Introduction}

Familial Mediterranean fever (FMF) is a disease characterized by recurrent attacks of peritonitis, pleuritis or arthritis accompanied by fever. Involvement of the reticuloendothelial system in FMF is hardly mentioned in the standard textbooks of medicine.$^{1,2}$ However, splenomegaly has been reported in as many as $57 \%$ of the cases in various series, ${ }^{3}$ and lymphadenopathy, both peripheral and abdominal, has been reported in 1 to $6 \% .^{4-9}$ The abdominal lymphadenopathy was always found in the mesentery during laparotomies for acute abdominal attacks of FMF. ${ }^{4-6}$ There has been a single case of retroperitoneal lymphadenopathy, found at autopsy, reported. ${ }^{6}$ We add here a second case of FMF with retroperitoneal lymphadenopthy, revealed by abdominal ultrasonography (US) and computerized tomography (CT). Biopsy of the glands, at exploratory laparotomy, showed non-specific hyperplasia. After an attack-free period of one year the enlarged lymph glands disappeared.

\section{Case report}

A 25 year old male patient was admitted to our department in 1986 for investigation of retroperitoneal space occupying lesions, revealed by abdominal ultrasound performed for recurrent loin pain. The urine sediment and intravenous urography (IVU) were normal. FMF was diagnosed at the age of 15 years. He had recurrent typical abdominal attacks, arthritis and pleuritis with fever. He took colchicine

Address requests for reprints to: Professor L. Cohen, M.D. Correspondence: D. Rimon, M.D.

Accepted: 8 March 1989 intermittently, and continued to have attacks of FMF. His parents immigrated from Kurdistan and his sister also had FMF. Physical examination was completely normal. Erythrocyte sedimentation rate was $40 \mathrm{~mm} /$ hour. His liver, kidney and thyroid function tests were normal, as well as blood count, serum amylase, uric acid, calcium, proteins and immunoglobulins. Alpha응 fetoprotein and beta-human chorionic gonadotropin in serum were negative. Recurrent serology waș negative for the following pathogens: cytomegalovirus, Epstein-Barr virus, human immunodeficiency virus, Chlamydia trachomatis, salmonella, brucella, Treponema pallidum and Toxoplasma gondii. Tests for antinuclear antibodies and rheumatoid factor were negative. Tuberculin skin test (5 units) was negative. Chest X-ray was normal. X-ray series of upper gastrointestinal tract, small bowel and large bowel with barium were negative. Rectal biopsy was negative for amyloidosis. Bone marrow biopsy was normal. Abdominal ultrasound done one month following the first examination, revealed paraaortic hypoechoic masses, $4 \mathrm{~cm}$ in diameter (Figure 1), at mid-abdominal levels without change from the previous examination. The liver, spleen, kidneys and pancreas were normal. Abdominal CT confirmed a retroperitoneal mass of low density consistent with enlarged paraaortic lymph nodes. The patient underwent an exploratory laparotomy, which revealed only enlarged paraaortic lymph glands. Other abdominal organs were normal. The liver and lymph glands were biopsied. Histological examination of the lymph glands showed reactive hyperplasia with sinus histiocytosis. Cultures for tuberculosis and fungi were negative. Liver biopsy was normal. Congo-red staining of all preparations was negative. The patient was discharged with an advice for permanent treatment with colchicine and 
a

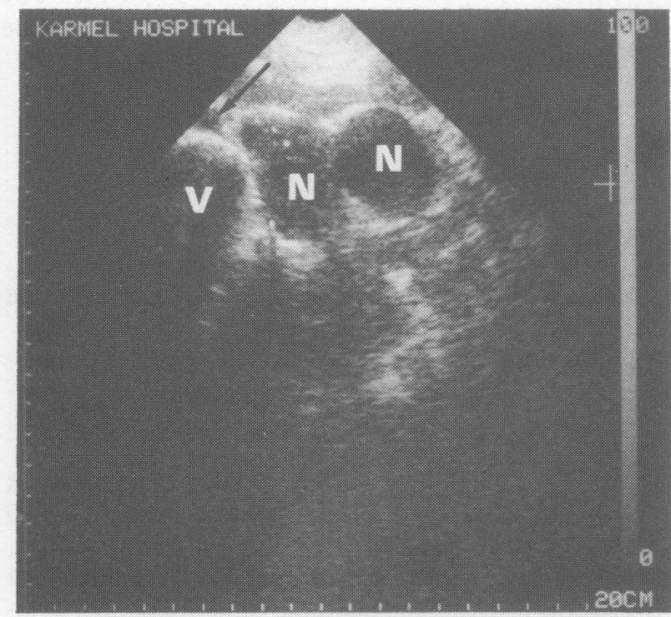

b

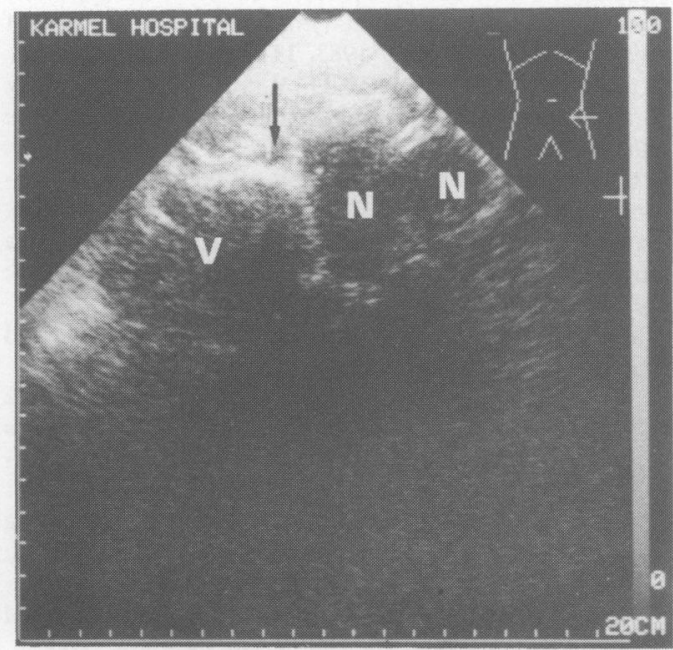

Figure 1 (a and b) Transverse ultrasound scan in umbilical level shows enlarged retroperitoneal masses $(\mathbf{N})$ in vicinity of a vertebra (V), consistent with enlarged lymph nodes. An arrow indicates the aorta.

for the last two years he has had no attacks of FMF. Ultrasound examination six months after the laparotomy still showed enlarged paraaortic lymph glands but the last examination done after one year showed a normal retroperitoneal space.

\section{Discussion}

Enlarged retroperitoneal, paraaortic lymph nodes are not usually inflammatory in origin, but are frequently due to neoplastic diseases. The neoplastic glands are either lymphoreticular neoplasms (non-Hodgkin lymphoma most commonly) or a lymphatic spread from primary tumours in the gastrointestinal tract, pancreas, genitalia, kidney, breast, lung, and malignant melanoma or sarcoma of the lower extremities. ${ }^{10,11}$ The inflammatory retroperitoneal, paraaortic lymph glands are secondary to either acute infections, such as enteric fever, abdominal abscess, infection secondary to viscus ulceration and/or perforation, or to chronic infections, such as tuberculosis and fungus infection..$^{10}$ Other rarely encountered causes for enlarged paraaortic, retroperitoneal lymph glands are syphilis, ${ }^{12}$ catscratch disease, ${ }^{13}$ infectious mononucleosis, ${ }^{14}$ lymphogranuloma venereum, ${ }^{15}$ AIDS-related complex, ${ }^{16}$ Whipple's disease, ${ }^{17}$ ankylosing spondylitis, ${ }^{15}$ systemic lupus erythematosus (SLE), ${ }^{15}$ rheumatoid arthritis, ${ }^{18}$ adult onset Still's disease, ${ }^{19}$ polyarteritis nodosa, ${ }^{15}$ sarcoidosis, ${ }^{14,15}$ pancreatitis ${ }^{20}$ and amyloidosis. ${ }^{21}$

Lymphadenopathy in patients with FMF has not been paid much attention. ${ }^{22-25}$ On the other hand Eliakim et al. $^{3}$ state that enlarged lymph nodes are often seen on laparotomy in patients with FMF. Searching the medical literature we found the following information: Reimann et al. ${ }^{4}$ report one patient with mesenteric lymphadenopathy out of 58 FMF patients $(1.7 \%)$. This patient was also one of a subgroup of 7 patients who underwent abdominal surgery and their pathological changes were documented $(14 \%)$. Siegal ${ }^{6}$ reports 3 patients with lymphadenopathy out of 50 FMF patients $(6 \%)$. The first patient had mesenteric lymphadenopathy and he was one of a subgroup of 11 patients who underwent exploratory laparotomy $(9 \%)$. The second patient had transient hilar lymphadenopathy, revealed by chest roentgenogram. The third patient had diffuse lymphadenopathy on palpation, mesenteric nodes at cholecystectomy, and preaortic nodes at autopsy. Schwabe and Peters ${ }^{8}$ report a patient with prolonged transient diffuse lymphadenopathy, with splenomegaly, arthritis and fever out of a series of 100 FMF patients $(1 \%)$. Armenian and Khachadurian ${ }^{7}$ report 7 patients with lymphadenitis out of 120 patients with FMF (6\%).

From the aforementioned data, it appears that: (1) lymphadenopathy occurs in $1-6 \%$ of FMF patients; (2) mesenteric lymphadenopathy can be found in $9-14 \%$ of FMF patients undergoing laparatomy during an abdominal attack of FMF; (3) peripheral and retroperitoneal lymphadenopathy rarely occurs in FMF. We have found only one case report of a patient with FMF, in whom retroperitoneal lymphadenopathy was found at autopsy. ${ }^{6}$ Our patient is therefore the first one to be diagnosed during life, by means of abdominal ultrasound and CT.

The histological diagnosis in every case reported was non-specific lymphoid hyperplasia. ${ }^{4,6,8}$ All biopsy 
and autopsy material was negative for amyloidosis. Splenomegaly was found in some of the FMF patients with lymphadenopathy. ${ }^{6,8}$ When lymphadenopathy is marked, or associated with splenomegaly, malignant

\section{References}

1. Wolff, S.M. Familial mediterranean fever (familial paroxysmal polyserositis). In: Braunwald, E., Isselbacher, K.J., Petersdorf, R.G., Wilson, J.D., Martin, J.B. \& Fauci, A.S. (eds) Harrison's Principles of Internal Medicine. McGraw-Hill, New York, 1987, pp. 1450-1452.

2. Wright, D.G. Familial mediterranean fever. In: Wyngaarden, J.B. \& Smith, L.H. (eds) Cecil Textbook of Medicine W.B. Saunders, Philadelphia, 1988, pp. 1196-1198.

3. Eliakim, M., Levy, M. \& Ehrenfeld, M. Recurrent Polyserositis. Elsevier/North-Holland, Amsterdam, 1981.

4. Reimann, H.A., Moadie, J., Semerdjian, S. \& Sahyoun, P.F. Periodic peritonitis - heredity and pathology. JAMA 1954, 154: 1254-1259.

5. Ehrenfeld, E.N., Eliakim, M. \& Rachmilewitz, M. Recurrent polyserositis (familial mediterranean fever; periodic disease). Am J Med 1961, 31: 107-123.

6. Siegal, S. Familial paroxysmal polyserositis. Am J Med 1964, 36: 893-918.

7. Armenian, H.K. \& Khachadurian, A.K. Familial paroxysmal polyserositis. Leb Med J 1973, 26: 605-614.

8. Schwabe, A.D. \& Peters, R.S. Familial mediterranean fever in Armenians. Analysis of 100 cases. Medicine 1974, 53: $453-462$.

9. Barakat, M.H., Karnik, A.M., Majeed, H.W.A., ElSobki, N.I. \& Fenech, F.F. Familial mediterranean fever (recurrent hereditary polyserositis) in Arabs - a study of 175 patients and review of the literature. $Q J \mathrm{Med} 1986$, 233: $837-847$.

10. Owens, A.H. Illustrative neoplastic diseases. In: Harvey, A.M., Johns, R.J., McKusick, V.A., Owens, A.H. \& Ross, R.S. (eds) The Principles and Practices of Medicine. Appleton-Century-Crofts, New York, 1980, pp. 617-631.

11. Jing, B., Wallace, S. \& Zornoza, J. Metastases to retroperitoneal and pelvic lymph nodes. Radiol Clin North Am 1982, 20: 511-530.

12. Bergstrom, J.F. \& Navin, J.J. Luetic lymphadenitis: lymphographic manifestations simulating lymphoma. Radiology 1973, 106: 287-288. lymphoma may have to be excluded by lymph node biopsy. Such cases are described by us and by Schwabe $\stackrel{\mathscr{Q}}{\circ}$ and Peters. ${ }^{8}$

13. Bitterman, H. \& Cohen, L. Retroperitoneal lymphadenopathy in cat-scratch disease. Harefuah 1985, 108: ه $21-23$.

14. Viamonte, M., Altman, D., Parks, R., Blum, E., Bevilacqua, M. \& Recher, L. Radiographic-pathologic correlation in the interpretation of lymphangioadenograms. Radiology 1963, 80: 903-916.

15. Rauste, J. Lymphographic findings in granulomatous inflammations and connective tissue diseases. Acta Radiol 1972, 317 (Suppl): 5-79.

16. Tirelli, U., Vaccher, E., Carbone, A., De Paoli, P. \& خ Morassut, S. Lymphangiography and abdominal computerized tomography in peristent generalized lymphadenopathy. AIDS Res 1986, 2: 149-153.

17. Graham, P.M., Kelly, C.R. \& Booth, J.A. Ultrasonic appearance of abdominal lymph nodes in a case of $\rightarrow$ Whipple's disease. JCU 1983, 11: 388-390.

18. Robertson, M.D.J., Hart, F.D., White, W.F., Nuki, G.\& Boardman, P.L. Rheumatoid lymphadenopathy. Ant Rheum Dis 1968, 27: 253-260.

19. Reginato, A.J., Schumacher, H.R., Baker, D.G., O'Co̊̂ิ $\overrightarrow{0}$ nor, C.R. \& Ferreiros, J. Adult onset Still's disease experience in 23 patients and literature review wit $\overline{\text { An }}$ emphasis on organ failure. Semin Arth Rheum 1987, 1 ? 39-57.

20. Dodd, G.D. Lymphography in diseases of the liver and pancreas. Radiol Clin North Am 1970, 8: 69-84.

21. Takebayashi, S., Ono, Y., Sakai, F., Tamura, S. \& Unayama, S. Computed tomography of amyloidosis involving retroperitoneal lymph nodes mimicking lymphoma. J Comput Assist Tomogr 1984, 8: 1025- 1027

22. Priest, R.J. \& Nixon, R.K. Familial recurring polyserositis: a disease entity. Ann Intern Med 1959, 51: 1253-1274.

23. Sohar, E., Gafni, J., Pras, M. \& Heller, H. Familial mediterranean fever. Am J Med 1967, 43: 227-253.

24. Ozer, F.L., Kaplaman, E. \& Zileli, S. Familial mediterranean fever in Turkey. Am J Med 1971, 50: 336-339.

25. Bakir, F. \& Murtadha, M. Periodic peritonitis. Report of 41 cases without amyloidosis. Trans $R$ Soc Trop Med Hyg 1975, 69: 111-117. 